\title{
Generation of polarized positron beams via collisions of ultrarelativistic electron beams
}

\author{
Huai-Hang Song $\odot,{ }^{1,3}$ Wei-Min Wang $\odot,,^{2,4, *}$ and Yu-Tong $\mathrm{Li}^{1,3,5,6, \dagger}$ \\ ${ }^{1}$ Beijing National Laboratory for Condensed Matter Physics, Institute of Physics, CAS, Beijing 100190, China \\ ${ }^{2}$ Department of Physics and Beijing Key Laboratory of Opto-electronic Functional Materials and Micro-nano Devices, \\ Renmin University of China, Beijing 100872, China \\ ${ }^{3}$ School of Physical Sciences, University of Chinese Academy of Sciences, Beijing 100049, China \\ ${ }^{4}$ Collaborative Innovation Center of IFSA, Shanghai Jiao Tong University, Shanghai 200240, China \\ ${ }^{5}$ Songshan Lake Materials Laboratory, Dongguan, Guangdong 523808, China \\ ${ }^{6}$ CAS Center for Excellence in Ultra-intense Laser Science, Shanghai 201800, China
}

(Received 19 March 2021; accepted 26 August 2021; published 13 September 2021)

\begin{abstract}
A scheme is proposed for generating a polarized positron beam via multiphoton Breit-Wheeler process during the collision of a $10-\mathrm{GeV}$-level, 20-pC seeding electron beam with a second $10-\mathrm{GeV}$, multi-nC driving electron beam. The high-density driving beam with the length and transverse size less than $1 \mu \mathrm{m}$ provides a strong self-generated field. The field experienced by the seeding beam can be unipolar by incorporating a proper impact parameter, which is crucial to realizing the positron polarization. We employ semiclassical Monte Carlo simulations to calculate the spin- and polarization-resolved photon emission and electron-positron pair production in the locally constant-field approximation. The simulation results show that a highly polarized positron beam with a polarization degree above $40 \%$ can be generated in several femtoseconds, which is robust against the beam parameters. The positron polarization degree can be further increased to $60 \%$ provided the seeding beam is initially polarized.
\end{abstract}

DOI: 10.1103/PhysRevResearch.3.033245

\section{INTRODUCTION}

Polarized positron beams are indispensable tools in many areas of science and technology. In surface physics, lowenergy polarized positron beams can be utilized to probe surface magnetism [1], current-induced spin polarization [2], and other spin phenomena at surfaces [3] in nondestructive ways. Complemented with polarized electron beams in the future electron-positron $\left(e^{-} e^{+}\right)$linear collider, high-energy polarized positron beams offer new prospects for stringently testing the Standard Model and efficiently suppressing unwanted background processes [4,5].

Low-energy and low-flux longitudinally polarized positrons can be produced from radioactive sources [6], but with large energy spreads and wide angular distributions. High-energy and initially unpolarized positrons can be directly polarized in the transverse direction in storage rings via radiative polarization [7,8] (so-called Sokolov-Ternov effect), in which the positron spin is gradually aligned with the magnetic field direction. However, the buildup time of the polarization in most facilities is long, typically at a time scale

\footnotetext{
*weiminwang1@ ruc.edu.cn

†ytli@iphy.ac.cn
}

Published by the American Physical Society under the terms of the Creative Commons Attribution 4.0 International license. Further distribution of this work must maintain attribution to the author(s) and the published article's title, journal citation, and DOI. of minutes to hours. In conventional accelerators, polarized positron beams are mainly achievable by the polarization transfer based on two steps. First a GeV-level unpolarized electron beam is passed through a helical undulator [9] or scattered off by a circularly polarized laser pulse [10] to emit circularly polarized $\gamma$ photons, and subsequently some of these $\gamma$ photons further convert into longitudinally polarized $e^{-} e^{+}$pairs in a high- $Z$ target via Bethe-Heitler $(\mathrm{BH})$ process. Another polarization transfer from electrons to positrons at $\mathrm{MeV}$ energies by polarized bremsstrahlung radiation is also demonstrated [11], with an efficiency of about $10^{-4}$ from electron to positron.

Compared with the $\mathrm{BH}$ process, polarized positrons could be more effectively generated via the multiphoton (or nonlinear) Breit-Wheeler (BW) process [12] in the strong-field quantum electrodynamics (QED) regime [13-16]. Producing abundant positrons or dense $e^{-} e^{+}$plasmas by means of stateof-the-art ultraintense lasers $[17,18]$ is extensively explored theoretically [19-21]. Unfortunately, these positrons are usually unpolarized due to the symmetrically oscillating field of common multicycle laser pulses [22,23]. Recently, several schemes are proposed to construct asymmetric fields for the transversely polarized positron generation, e.g., one can employ elliptically polarized [24] or two-color linearly polarized [25] laser pulses. Longitudinally polarized positrons can also be produced via the helicity transfer from initially longitudinally polarized electrons in ultraintense circularly polarized laser fields [26]. Alternatively, the strong self-generated fields of high-energy, high-density electron beams in beam-beam collisions based on conventional accelerators can also trigger 


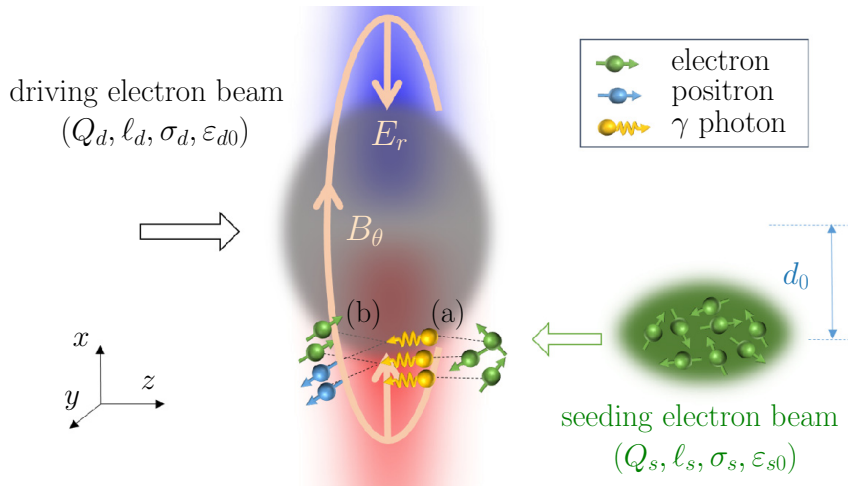

FIG. 1. A schematic of generating polarized positron beams via the collision of two electron beams. An ultrarelativistic seeding beam collides head-on with an ultrarelativistic, high-density driving beam. A suitable impact parameter $d_{0}$, defined as the perpendicular distance between two beam propagating axes, results in a strong unipolar field experienced by the seeding beam. There are two-step QED processes in the interaction: (a) electrons and positrons emit $\gamma$ photons via multiphoton beamstrahlung; (b) $\gamma$ photons decay into polarized $e^{-} e^{+}$ pairs via multiphoton $\mathrm{BW}$ process.

various QED processes [27], including multiphoton beamstrahlung [28-30], multiphoton BW [31,32], and even fully nonperturbative processes [33]. Whether one can obtain highenergy polarized positrons via the multiphoton $\mathrm{BW}$ process from beam-beam collisions demands studying.

In this paper, we put forward a scheme for the generation of transversely polarized positron beams based on the collision of two electron beams. A high-density, ultrarelativistic driving electron beam (total charge of several $\mathrm{nC}$, beam length, and transverse size less than $1 \mu \mathrm{m}$ ) provides the strong selfgenerated field, and a second ultrarelativistic seeding electron beam (kinetic energy of $10-\mathrm{GeV}$ level) with the smaller transverse size travels through part of the field for the $\gamma$ photon emission and the polarized $e^{-} e^{+}$pair production, as sketched in Fig. 1. The driving beam parameters can be expected at the FACET II facility [34] by means of advanced techniques [35,36], plasma lens [37], or beam-multifoil interaction [38]. The self-generated field of the driving beam, composed of the radial electric component and azimuthal magnetic component (of the order of $10^{5} \mathrm{~T}$ ), is nonoscillating. By adjusting the impact parameter between two beams properly, the seeding beam can only undergo a field that is unipolar. Hence, the magnetic field in the rest frame of the seeding beam is approximately along one direction, which is a key factor for the spin polarization of both primary electrons of the seeding beam and the newly generated $e^{-} e^{+}$pairs. With $e^{-} e^{+}$spin and $\gamma$-photon polarization effects considered, our simulations demonstrate a highly polarized positron beam with an average polarization above $40 \%$ or $60 \%$ can be obtained if an initially unpolarized or specifically polarized seeding beam is employed.

This paper is structured as follows. Section II presents the characteristics of the self-generated field of the driving beam, and the adopted theoretical model for the calculation of spin- and polarization-resolved photon emission and $e^{-} e^{+}$ pair production. In Sec. III, we analyze the simulation results and investigate the influence of two beam parameters on the positron yield and positron polarization in detail. In addition, a scheme for further improving the positron polarization by employing an initially polarized seeding beam is proposed. Finally, a brief conclusion is drawn in Sec. IV.

\section{THEORETICAL MODEL AND SIMULATION METHOD}

Here, we consider a high-density driving electron beam propagating along the $+z$ axis, with a bi-Gaussian charge density profile,

$$
\rho=\rho_{0} e^{-r^{2} / 2 \sigma_{d}^{2}} e^{-(z-z 0-v t)^{2} / 2 \ell_{d}^{2}},
$$

where $\rho_{0}=\frac{Q_{d}}{(2 \pi)^{3 / 2} \sigma_{d}^{2} \ell_{d}}$ is the peak charge density, $Q_{d}$ is the total charge, $\ell_{d}$ is the bunch length, $\sigma_{d}$ is the transverse size, $v \approx c$ is the beam velocity, $z_{0}$ is the initial position at the initial time $t=0, c$ is the speed of light in vacuum, and $r=\sqrt{x^{2}+y^{2}}$. For an ultrarelativistic electron beam of Lorentz factor $\gamma_{d}=1 / \sqrt{1-v^{2} / c^{2}} \gg 1$, the self-generated field around the beam is composed of the radial electric field $E_{r}$ and the azimuthal magnetic field $B_{\theta}$, which can be well approximated as $[28,38]$

$$
\begin{aligned}
E_{r}(r, z, t) & \approx B_{\theta}(r, z, t) \\
& \approx 4 \pi \rho_{0} \frac{\sigma_{d}^{2}}{r}\left(1-e^{-r^{2} / 2 \sigma_{d}^{2}}\right) e^{-\left(z-z_{0}-v t\right)^{2} / 2 \ell_{d}^{2}} .
\end{aligned}
$$

Longitudinal field components almost vanish, i.e., $E_{z} \approx 0$ and $B_{z} \approx 0$. At $r=r_{m} \approx 1.585 \sigma_{d}$ and $z=z_{0}+v t, B_{\theta}$ has a maximum strength

$$
B_{\theta}^{\max } \approx 1.08 \times 10^{4} \times \frac{Q_{d}(\mathrm{nC})}{\sigma_{d}(\mu \mathrm{m}) \times \ell_{d}(\mu \mathrm{m})}(\mathrm{T}) .
$$

If the seeding electron beam with an initial kinetic energy $\varepsilon_{s 0}$ collides head-on with the driving electron beam, the maximum of the quantum parameter $\chi_{e}=\left(|e| \hbar / m_{e}^{3} c^{4}\right)\left|F_{\mu \nu} p^{\nu}\right|$ during the interaction is

$$
\chi_{e}^{\max } \approx 0.0096 \times \frac{Q_{d}(\mathrm{nC}) \times \varepsilon_{s 0}(\mathrm{GeV})}{\sigma_{d}(\mu \mathrm{m}) \times \ell_{d}(\mu \mathrm{m})},
$$

where $F_{\mu \nu}$ is the field tensor, $p^{\nu}$ is the electron fourmomentum, $\hbar$ is the reduced Planck constant, $m_{e}$ is the electron mass, and $e$ is the electron charge, respectively.

During the collision of two beams, the photon emission and pair production with quantum stochastic nature are processed using the common Monte Carlo algorithms [39-41], but with spin- and polarization-resolved probabilities $[14,26,42,43]$. The classical dynamics of electrons and positrons in the external electromagnetic field are described by Newton-Lorentz equations, and the spin precession is calculated according to the Thomas-Bargmann-Michel-Telegdi equation [44].

The photon emission probability is derived based on the locally constant-field approximation (LCFA) and written as $[14,26,43]$

$$
\begin{aligned}
\frac{d^{2} W_{\gamma}}{d u d t}= & \frac{\alpha m_{e}^{2} c^{4}}{4 \sqrt{3} \pi \hbar \varepsilon_{e}}\left\{\frac{u^{2}-2 u+2}{1-u} K_{2 / 3}(y)-\operatorname{Int} K_{1 / 3}(y)\right. \\
& -u K_{1 / 3}(y)\left(\boldsymbol{S}_{i} \cdot \boldsymbol{e}_{2}\right)-\frac{u}{1-u} K_{1 / 3}(y)\left(\boldsymbol{S}_{f} \cdot \boldsymbol{e}_{2}\right) \\
& +\left[2 K_{2 / 3}(y)-\operatorname{Int} K_{1 / 3}(y)\right]\left(\boldsymbol{S}_{i} \cdot \boldsymbol{S}_{f}\right)
\end{aligned}
$$




$$
\begin{aligned}
& +\frac{u^{2}}{1-u}\left[K_{2 / 3}(y)-\operatorname{Int} K_{1 / 3}(y)\right]\left(\boldsymbol{S}_{i} \cdot \boldsymbol{e}_{v}\right)\left(\boldsymbol{S}_{f} \cdot \boldsymbol{e}_{v}\right) \\
& +\frac{u}{1-u} K_{1 / 3}(y)\left(\boldsymbol{S}_{i} \cdot \boldsymbol{e}_{1}\right) \xi_{1} \\
& +\left[\frac{2 u-u^{2}}{1-u} K_{2 / 3}(y)-u \operatorname{Int} K_{1 / 3}(y)\right]\left(\boldsymbol{S}_{i} \cdot \boldsymbol{e}_{v}\right) \xi_{2} \\
& \left.+\left[K_{2 / 3}(y)-\frac{u}{1-u} K_{1 / 3}(y)\left(\boldsymbol{S}_{i} \cdot \boldsymbol{e}_{2}\right)\right] \xi_{3}\right\},
\end{aligned}
$$

where $K_{v}(y)$ is the modified Bessel function of the second kind with a noninteger $v, \operatorname{Int} K_{1 / 3}(y) \equiv \int_{y}^{\infty} K_{1 / 3}(x) d x, y=$ $2 u /\left[3(1-u) \chi_{e}\right], u=\varepsilon_{\gamma} / \varepsilon_{e}, \varepsilon_{e}$ is the electron energy before emitting the photon, $\varepsilon_{\gamma}$ is the emitted photon energy, and $\alpha \approx 1 / 137$ is the fine structure constant. Spin vectors $\boldsymbol{S}_{i}$ and $\boldsymbol{S}_{f}$ correspond to spin states of the electron before and after the photon emission, respectively, with the norm $\left|\boldsymbol{S}_{i, f}\right|=1$. The three-dimensional (3D) spin information is completely preserved $[26,42]$ when calculating the radiative polarization of the electron. The Stokes parameters $\xi=\left(\xi_{1}, \xi_{2}, \xi_{3}\right)$ of the emitted photon in the basis $\left(\boldsymbol{e}_{1}, \boldsymbol{e}_{2}, \boldsymbol{e}_{v}\right)$ is determined via the method adopted in [43], where $\boldsymbol{e}_{v}$ is the unit vector along the photon velocity, $\boldsymbol{e}_{1}$ is the unit vector along the transverse acceleration of its parent electron, and $\boldsymbol{e}_{2}=\boldsymbol{e}_{v} \times \boldsymbol{e}_{1}$.

The positron spin vector $S_{+}$at the moment of production can be determined by the pair production probability $[14,26]$ :

$$
\begin{aligned}
\frac{d^{2} W_{ \pm}}{d \varepsilon_{+} d t}= & \frac{\alpha m_{e}^{2} c^{4}}{2 \sqrt{3} \pi \hbar \varepsilon_{\gamma}^{2}}\left\{\left[\frac{\varepsilon_{+}^{2}+\varepsilon_{-}^{2}}{\varepsilon_{+} \varepsilon_{-}}-\xi_{3}^{\prime}\right] K_{2 / 3}(y)+\operatorname{Int} K_{1 / 3}(y)\right. \\
& -\xi_{1}^{\prime} \frac{\varepsilon_{\gamma}}{\varepsilon_{-}} K_{1 / 3}(y)\left(\boldsymbol{S}_{+} \cdot \boldsymbol{e}_{1}^{\prime}\right) \\
& +\xi_{2}^{\prime}\left[\frac{\varepsilon_{\gamma}}{\varepsilon_{+}} \operatorname{Int} K_{1 / 3}(y)+\frac{\varepsilon_{+}^{2}-\varepsilon_{-}^{2}}{\varepsilon_{+} \varepsilon_{-}} K_{2 / 3}(y)\right]\left(\boldsymbol{S}_{+} \cdot \boldsymbol{e}_{v}\right) \\
& \left.-\left(\frac{\varepsilon_{\gamma}}{\varepsilon_{+}}-\xi_{3}^{\prime} \frac{\varepsilon_{\gamma}}{\varepsilon_{-}}\right) K_{1 / 3}(y)\left(\boldsymbol{S}_{+} \cdot \boldsymbol{e}_{2}^{\prime}\right)\right\},
\end{aligned}
$$

where $y=2 \varepsilon_{\gamma}^{2} /\left(3 \chi_{\gamma} \varepsilon_{+} \varepsilon_{-}\right), \varepsilon_{-}, \varepsilon_{+}$, and $\varepsilon_{\gamma}$ are the energies of the newly produced electron and positron, and annihilated $\gamma$ photon, respectively. Another quantum parameter $\chi_{\gamma}=$ $\left(|e| \hbar^{2} / m_{e}^{3} c^{4}\right)\left|F_{\mu \nu} k^{\nu}\right|$ characterizes the importance of the pair production, with $\hbar k^{\nu}$ being the photon four-momentum. The photon Stokes parameters $\xi^{\prime}=\left(\xi_{1}^{\prime}, \xi_{2}^{\prime}, \xi_{3}^{\prime}\right)$ in Eq. (6) are defined in the basis $\left(\boldsymbol{e}_{1}^{\prime}, \boldsymbol{e}_{2}^{\prime}, \boldsymbol{e}_{v}\right)$, where $\boldsymbol{e}_{1}^{\prime}$ is the unit vector along the transverse positron acceleration $\boldsymbol{E}+\boldsymbol{e}_{v} \times \boldsymbol{B}-\boldsymbol{e}_{v} \cdot\left(\boldsymbol{e}_{v}\right.$. $\boldsymbol{E}), \boldsymbol{e}_{2}^{\prime}=\boldsymbol{e}_{v} \times \boldsymbol{e}_{1}^{\prime}$ is the unit vector along the magnetic field in the positron rest frame, and $\boldsymbol{E}$ and $\boldsymbol{B}$ are electric and magnetic fields at the photon annihilation position, respectively. For the scenario considered here, vectors $\boldsymbol{e}_{1}^{\prime}$ and $\boldsymbol{e}_{2}^{\prime}$ are almost unchanged for each $\gamma$ photon during passing through the strong self-generated field of the driving beam, since the emitted $\gamma$ photons are well collimated along the $-z$ axis. Therefore, the Stokes parameters of each photon required in Eq. (6) nearly keep constant at different times, and are also identical to that in Eq. (5), i.e., $\xi^{\prime} \approx \xi$. The small difference is ignored in our analysis for simplicity although it is exactly captured in our simulations.

Analogically, the approach described above can also be applied to the radiative polarization of positrons and the deter- mination of spin vector $\boldsymbol{S}_{-}$of newly produced electrons [26]. The correlation terms of $\boldsymbol{S}_{f}$ and $\boldsymbol{\xi}$ are ignored in Eq. (5) since they are calculated separately in the Monte Carlo methods employed by us $[42,45]$. Similarly, we have also ignored the terms involving both $\boldsymbol{S}_{+}$and $\boldsymbol{S}_{-}$in Eq. (6).

For the widely studied cases with laser fields, LCFA is generally considered to be valid under the strong-field condition of $a_{0}=|e| E_{0} /\left(m_{e} c \omega_{0}\right) \gg 1$ [15,46-49], where $\omega_{0}$ and $E_{0}$ are the laser frequency and field amplitude, respectively. The self-generated field of the driving electron beam can be approximately treated as the half-cycle laser field with a wavelength of $4 \ell_{d}$. Therefore, the equivalent validity condition of LCFA is $a_{0}^{*} \approx 2|e| E_{r}^{\max } \ell_{d} / \pi m_{e} c^{2} \gg 1$, i.e.,

$$
a_{0}^{*} \approx 4 \times \frac{Q_{d}(\mathrm{nC})}{\sigma_{d}(\mu \mathrm{m})} \gg 1 .
$$

\section{SIMULATION RESULTS AND ANALYSIS}

\section{A. Simulation setups}

To investigate the generation of polarized positrons in the collision of two ultrarelativistic electron beams, 3D Monte Carlo simulations are performed. The self-generated field of the driving beam is implemented by the analytic expression according to Eq. (2), while that of the low-density seeding beam is much weaker and therefore can be ignored (just of the order of $10^{2} \mathrm{~T}$ ). We take the driving electron beam with $Q_{d}=5 \mathrm{nC}, \ell_{d}=0.3 \mu \mathrm{m}, \sigma_{d}=0.5 \mu \mathrm{m}$, and the initial kinetic energy $\varepsilon_{d 0}=10 \mathrm{GeV}$. Note that according to Eq. (2), $\varepsilon_{d 0}$ does not affect its self-generated field as long as the precondition $\gamma_{d} \gg 1$ holds. The other initially unpolarized seeding electron beam propagating along the $-z$ axis has the total charge $Q_{s}=20 \mathrm{pC}$ (consisting of $N_{s} \approx 1.25 \times 10^{8}$ primary electrons), bunch length $\ell_{s}=1.0 \mu \mathrm{m}$, transverse size $\sigma_{s}=0.25 \mu \mathrm{m}, \varepsilon_{s 0}=15 \mathrm{GeV}$, and energy spread $\Delta \varepsilon_{s 0} / \varepsilon_{s 0}=$ 0.01 , which can be provided by conventional accelerators or via future wakefield accelerations [34,50]. There is a nonzero impact parameter $d_{0}=0.8 \mu \mathrm{m}$ between two beams, i.e., $d_{0} \approx$ $r_{m}$. At the beginning $t=0$, the driving and seeding beams are centered at $\left(x_{0}, y_{0}, z_{0}\right)=(0,0,-2 \mu \mathrm{m})$ and $\left(-d_{0}, 0,2 \mu \mathrm{m}\right)$, respectively. For the chosen parameters, the maximum of quantum parameter $\chi_{e}^{\max }$ during the interaction is about 4.8 , implying the interaction enters the QED regime, in which multiphoton BW process can be important. Meanwhile, the normalized field strength $a_{0}^{*} \approx 40 \gg 1$ ensures the validity of LCFA.

\section{B. Simulation results}

We first show some essential properties of generated positrons in Fig. 2, and then present a detailed analysis of twostep processes to explain the observed positron polarization effect in Fig. 3.

Figure 2(a) shows the transverse angular distribution of positron number density $d^{2} N_{+} / d \theta_{x} d \theta_{y}$ after the collision. The generated positrons are mainly propagating along the initial seeding beam velocity ( $-z$ axis) with an angular divergence around $10 \mathrm{mrad}$ (full width at half maximum). The total positron number $N_{+}$is about $4 \times 10^{5}$, i.e., $N_{+} / N_{s} \approx 3.2 \times$ $10^{-3}$. After being generated, positrons are deflected along the $+x$ axis by the strong field of the driving beam. Lower-energy 

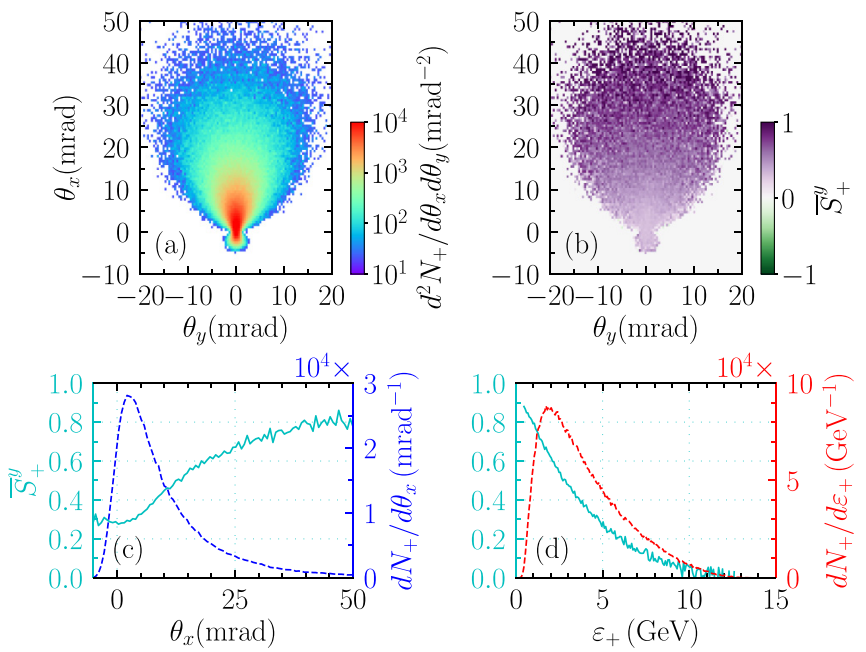

FIG. 2. Properties of the generated positrons after the collision. Transverse angular distributions of (a) positron number density $d^{2} N_{+} / d \theta_{x} d \theta_{y}$ and (b) positron polarization $\bar{S}_{+}^{y}$ vs deflection angles $\theta_{x}$ and $\theta_{y}$, where $\theta_{x(y)}=\arctan \left[p_{x(y)} /\left|p_{z}\right|\right]$. (c) $\bar{S}_{+}^{y}$ (cyan solid) and $d N_{+} / d \theta_{x}$ (blue dashed) vs $\theta_{x}$. (d) $\bar{S}_{+}^{y}$ (cyan solid) and positron spectrum $d N_{+} / d \varepsilon_{+}$(red dashed) vs positron energy $\varepsilon_{+}$.

positrons have larger deflection angles $\theta_{x}$ due to smaller relativistic masses. As a key result, the generated positrons appear to be transversely polarized, as shown by the corresponding positron polarization $\bar{S}_{+}^{y}$ (average positron spin) in Fig. 2(b). The polarization degree of all generated positrons is about 0.43 , and its positive value denotes that the positron spins are predominantly oriented along the $+y$ axis, which is the direction of the magnetic field in the positron rest frame. Positron polarization $\bar{S}_{+}^{y}$ increases with the increase of $\theta_{x}$, but at the same time the positron number density $d N_{+} / d \theta_{x}$ dramatically declines, as shown in Fig. 2(c). More specifically, at the larger angle $\theta_{x}>20 \mathrm{mrad}, \bar{S}_{+}^{y}$ can exceed 0.6 ; however,
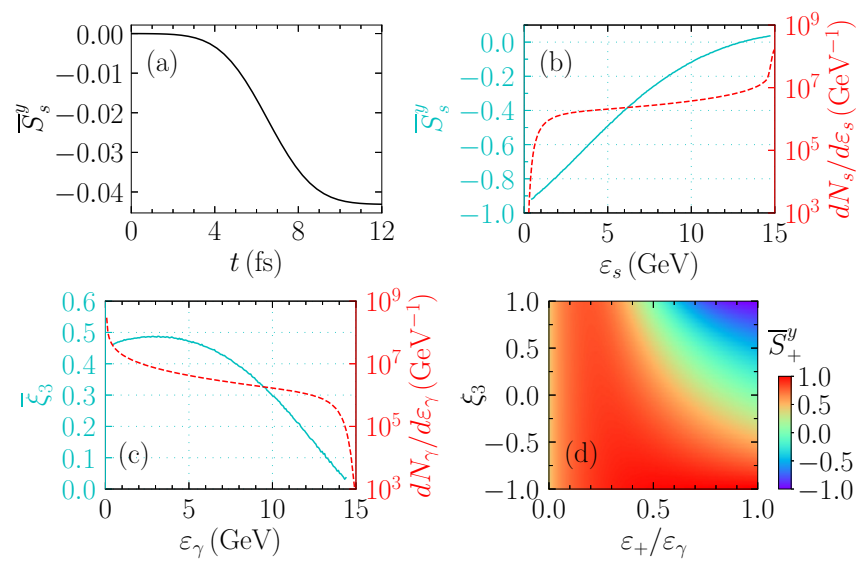

FIG. 3. (a) Evolution of polarization degree of the seeding beam. (b) Electron spectrum $d N_{s} / d \varepsilon_{s}$ (red dashed) and electron polarization $\bar{S}_{s}^{y}$ (cyan solid) vs electron energy $\varepsilon_{s}$ of the seeding beam. (c) Photon spectrum $d N_{\gamma} / d \varepsilon_{\gamma}$ (red dashed) and photon polarization $\bar{\xi}_{3}$ (cyan solid) vs photon energy $\varepsilon_{\gamma}$. (d) Theoretical positron polarization $\bar{S}_{+}^{y}$ vs photon polarization $\xi_{3}$ and the energy ratio $\varepsilon_{+} / \varepsilon_{\gamma}$ with $\chi_{\gamma}=1$. at $\theta_{x}<10 \mathrm{mrad}$ where most positrons are concentrated, $\bar{S}_{+}^{y}$ is less than 0.4. In Fig. 2(d), it clearly demonstrates that $\bar{S}_{+}^{y}$ is strongly dependent on the positron energy $\varepsilon_{+}$. For relatively low-energy positrons of $1 \mathrm{GeV}$, their $\bar{S}_{+}^{y}$ is about 0.8 , while $\bar{S}_{+}^{y}$ is only about 0.1 for high-energy positrons of $10 \mathrm{GeV}$. The properties of generated electrons are similar to those of positrons, but with the opposite deflection angle (along the $-x$ axis) and opposite polarization direction (along the $-y$ axis).

For generating polarized positrons, the first step is the photon emission by the seeding beam in the strong unipolar field supplied by the driving beam. As shown in Fig. 3(a), the initially unpolarized seeding beam can be directly polarized as emitting $\gamma$ photons via radiative polarization. However, its final polarization degree is limited to $\bar{S}_{s}^{y} \approx-0.04$, much weaker than 0.43 of the generated positrons. This is because the radiative polarization is a weaker spin-dependent process compared with the positron polarization via the nonlinear BW process [25]. Therefore, the former needs more polarization time, and the ultrashort interaction time $\sim \ell_{d} / c \approx 1 \mathrm{fs}$ limits it severely. Figure 3(b) illustrates the dependence of the electron polarization $\bar{S}_{s}^{y}$ on the electron energy $\varepsilon_{s}$ of the seeding beam. Lower-energy electrons possess higher polarization since the electron spin is more likely to flip to the direction antiparallel to the magnetic field in its rest frame (i.e., along the $-y$ axis) only when a high-energy photon is emitted according to Eq. (5) [51]. The spin-flip electron simultaneously loses a substantial fraction of its energy.

The emitted photons are highly polarized, as presented in Fig. 3(c). The positive value of photon polarization $\bar{\xi}_{3}$ (average Stokes parameter) indicates that these photons are mainly linearly polarized along the $x$ axis. Furthermore, $\bar{\xi}_{3}$ is closely related to the photon energy $\varepsilon_{\gamma}$, in which $\bar{\xi}_{3}$ of higher-energy photons is generally smaller. This is attributed to the spin- and polarization-dependent photon emission governed by Eq. (5). The polarization $\bar{\xi}_{3}$ of high-energy photons is strongly suppressed since the high-energy electrons that emit them are weakly polarized. In contrast, $\bar{\xi}_{3}$ of low-energy photons is insensitive to spin states of their parent electrons and always positive [45].

Let us analyze the positron polarization during the second step that $\gamma$ photons annihilate into $e^{-} e^{+}$pairs. As discussed above, the primary electrons of the seeding beam or generated electrons/positrons can only be transversely polarized, and consequently the emitted photons are mainly linearly polarized along the $x$ axis, i.e., $\bar{\xi}_{1} \approx 0, \bar{\xi}_{2} \approx 0$, and $\bar{\xi}_{3} \neq 0$. For this reason, based on the pair production probability of Eq. (6), the positron polarization $\bar{S}_{+}^{y}$ can be simplified as

$$
\bar{S}_{+}^{y}=\frac{\varepsilon_{\gamma}\left(1 / \varepsilon_{+}-\xi_{3} / \varepsilon_{-}\right) K_{1 / 3}(y)}{\left[\varepsilon_{+} / \varepsilon_{-}+\varepsilon_{-} / \varepsilon_{+}-\xi_{3}\right] K_{2 / 3}(y)+\operatorname{Int} K_{1 / 3}(y)} .
$$

According to Eq. (8), the theoretical $\bar{S}_{+}^{y}$ as a function of the parent photon polarization $\xi_{3}$ and the energy ratio $\varepsilon_{+} / \varepsilon_{\gamma}$ is plotted in Fig. 3(d). It can be seen that $\bar{S}_{+}^{y}$ of high-energy positrons is rather sensitive to $\xi_{3}$, while it is insensitive for low-energy positrons. Moreover, as high-energy $\gamma$ photons are weakly polarized, e.g., $\bar{\xi}_{3}<0.2$ for $\varepsilon_{\gamma}>12 \mathrm{GeV}$ as displayed in Fig. 3(c), one can deduce that high-energy positrons have relatively small $\bar{S}_{+}^{y}$ from Fig. 3(d), which is consistent with the simulation results shown in Fig. 2(d). Noticeably, 


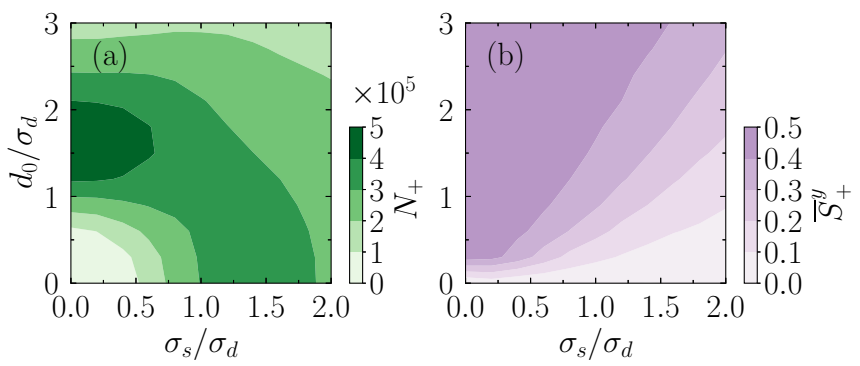

FIG. 4. (a) Positron number $N_{+}$and (b) positron polarization $\bar{S}_{+}^{y}$ vs $\sigma_{s} / \sigma_{d}$ and $d_{0} / \sigma_{d}$, where $\sigma_{d}$ is fixed at $0.5 \mu \mathrm{m}$ and other parameter are the same as those in Fig. 2.

if intermediate $\gamma$ photons possess negative $\bar{\xi}_{3}$ (dominantly linearly polarized along the $y$ axis), the polarization degree of their generated positrons will be significantly improved since $\bar{S}_{+}^{y}$ is always positive at both low- and high-energy regions. This can be achieved by employing an initially polarized seeding beam, which will be discussed below in more detail.

\section{Influence of two beam parameters}

We proceed to investigate the influence of beam parameters on the positron number $N_{+}$and the polarization degree $\bar{S}_{+}^{y}$. To gain a highly polarized positron beam, the field experienced by the seeding beam should be unipolar. In this respect, the seeding beam should keep away from the center of the driving beam. On the other hand, one needs to make sure that as many positrons as possible are generated. Therefore, there is an optimal impact parameter $d_{0}$ between two beams for a given transverse size $\sigma_{s}$ of the seeding beam. The achievable $N_{+}$and $\bar{S}_{+}^{y}$ as a function of $d_{0} / \sigma_{d}$ and $\sigma_{s} / \sigma_{d}$ are presented in Figs. 4(a) and 4(b), respectively, where the transverse size of the driving beam $\sigma_{d}$ is fixed at $0.5 \mu \mathrm{m}$ as before. In general, the colliding condition of $\sigma_{s}=0.5 \sigma_{d}$ and $d_{0}=1.5 \sigma_{d}$ is optimal, which can simultaneously ensure appreciable $N_{+}$and $\bar{S}_{+}^{y}$ under a relaxed requirement for $\sigma_{s}$. Further decreasing $\sigma_{s}$ does not significantly increase $N_{+}$or $\bar{S}_{+}^{y}$. In practice, if one cannot ensure this optimal condition, a larger $d_{0}$ should be adopted to meet a larger $\sigma_{s}$ for $\sigma_{s} / \sigma_{d}>0.5$. In this way, although $N_{+}$ decreases, $\bar{S}_{+}^{y}$ can be kept nearly unchanged around 0.4.

Then, in Figs. 5(a) and 5(b), the roles of bunch length $\ell_{d}$ and transverse size $\sigma_{d}$ of the driving beam are investigated, under the optimal colliding condition obtained above and with a fixed total charge $Q_{d}=5 \mathrm{nC}$. For each $\sigma_{d}, N_{+}$increases exponentially with the decrease of $\ell_{d}$ for $\ell_{d}>0.1 \mu \mathrm{m}$, while $\bar{S}_{+}^{y}$ decreases only from 0.45 to 0.4 . Hence, compressing the driving beam in this parameter range is a very effective way to improve the yield of polarized positrons, due to the enhancement of the self-generated field, and consequently quantum parameter $\chi_{e}^{\max }$. For a highly compressed driving beam of $\ell_{d}=0.1 \mu \mathrm{m}$ and $\sigma_{d}=0.2 \mu \mathrm{m}$, the positron generation efficiency can reach $N_{+} / N_{s} \sim 0.1$. However, $N_{+}$substantially drops with further decreasing $\ell_{d}$ for $\ell_{d}<0.02 \mu \mathrm{m}$ as the collision time is rather short. The positron mitigation regime of the tightly compressed beam collision [33] can be qualitatively explained by $N_{\gamma} \sim W_{\gamma} \ell_{d} / c \propto \ell_{d}^{1 / 3}$ and $N_{+} \sim$ $N_{\gamma} W_{ \pm} \ell_{d} / c \propto \ell_{d}^{2 / 3}$ in the limit $\chi_{e} \gg 1$.
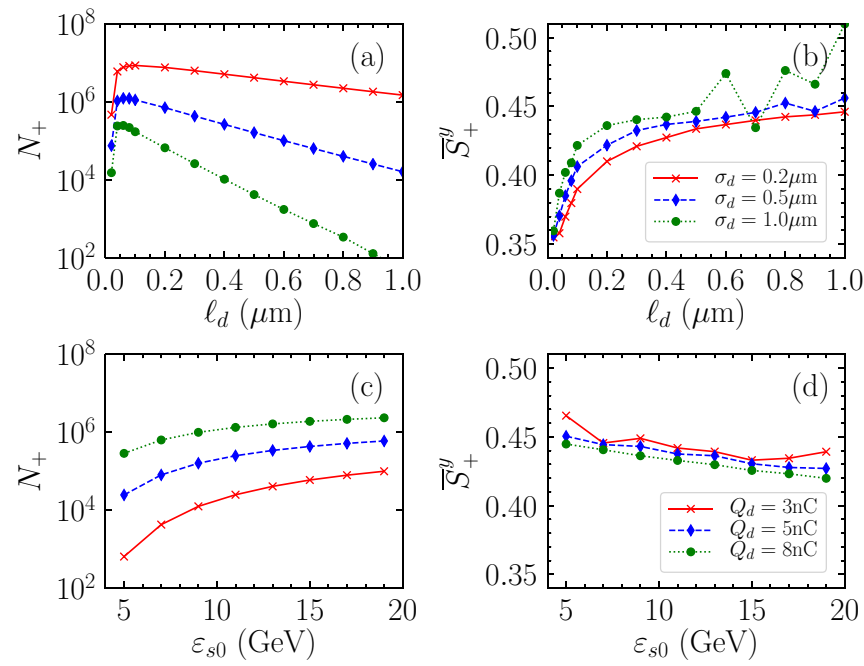

FIG. 5. Top row: (a) positron number $N_{+}$and (b) positron polarization $\bar{S}_{+}^{y}$ vs $\ell_{d}$ in three cases of $\sigma_{d}=0.2 \mu \mathrm{m}$ (red solid), $\sigma_{d}=$ $0.5 \mu \mathrm{m}$ (blue dashed), and $\sigma_{d}=1.0 \mu \mathrm{m}$ (green dotted), respectively. Bottom row: (c) $N_{+}$and (d) $\bar{S}_{+}^{y}$ vs $\varepsilon_{s 0}$ in three cases of $Q_{d}=3 \mathrm{nC}$ (red solid), $Q_{d}=5 \mathrm{nC}$ (blue dashed) and $Q_{d}=8 \mathrm{nC}$ (green dotted), respectively. The more obvious fluctuations of $\bar{S}_{+}^{y}$ for green dotted line of (b) and red solid line of (d) comes from quantum stochastic effects due to small number of positrons. Other parameters are same as those in Fig. 2.

In addition, increasing the driving beam charge $Q_{d}$ or the seeding beam energy $\varepsilon_{s 0}$ can also dramatically increase $N_{+}$, as shown in Figs. 5(c) and 5(d). $N_{+}$is increased by four orders of magnitude from $Q_{d}=3 \mathrm{GeV}$ and $\varepsilon_{s 0}=5 \mathrm{nC}$ to $Q_{d}=8$ $\mathrm{nC}$ and $\varepsilon_{s 0}=20 \mathrm{GeV}$, while the decrease of $\bar{S}_{+}^{y}$ is ignorable. For the former set of parameters, $\chi_{e}^{\max }$ is only 1 ; while for the latter, $\chi_{e}^{\max }$ is up to 7.6. In general, further compressing the driving beam size [35-38] (before reaching the mitigation regime) or increasing $Q_{d}$ or $\varepsilon_{s 0}$ can increase the positron yield effectively, meanwhile ensuring a high polarization degree of positrons, provided the optimal colliding condition of $\sigma_{s}=$ $0.5 \sigma_{d}$ and $d_{0}=1.5 \sigma_{d}$ is fulfilled.

\section{Improving positron polarization by initially polarized seeding beam}

As mentioned above, the polarization of high-energy positrons highly relies on the polarization of their parent $\gamma$ photons [see Fig. 3(d)], and the latter depends on the initial polarization of the seeding beam. For an unpolarized seeding beam of $\bar{S}_{s 0}^{y}=0$, the emitted $\gamma$ photons will carry positive $\bar{\xi}_{3}$, which decreases with the increase of the photon energy $\varepsilon_{\gamma}$ [see Fig. 3(c)]. To further improve the positron polarization, especially in the high-energy range, negative $-\bar{\xi}_{3}$ photons are required. Here, we propose to employ an initially polarized seeding beam [52-55] with a positive value of the transverse polarization $\bar{S}_{s 0}^{y}>0$ to achieve it. We first take a $100 \%$ polarized seeding beam of $\bar{S}_{s 0}^{y}=1$, whose spin vectors are directed parallel to the magnetic field in the rest frame of the seeding beam. Under this condition, the transverse angular distribution of the positron polarization $\bar{S}_{+}^{y}$ after the collision is shown in Fig. 6(a). Compared with the unpolarized case in Fig. 2(b), 

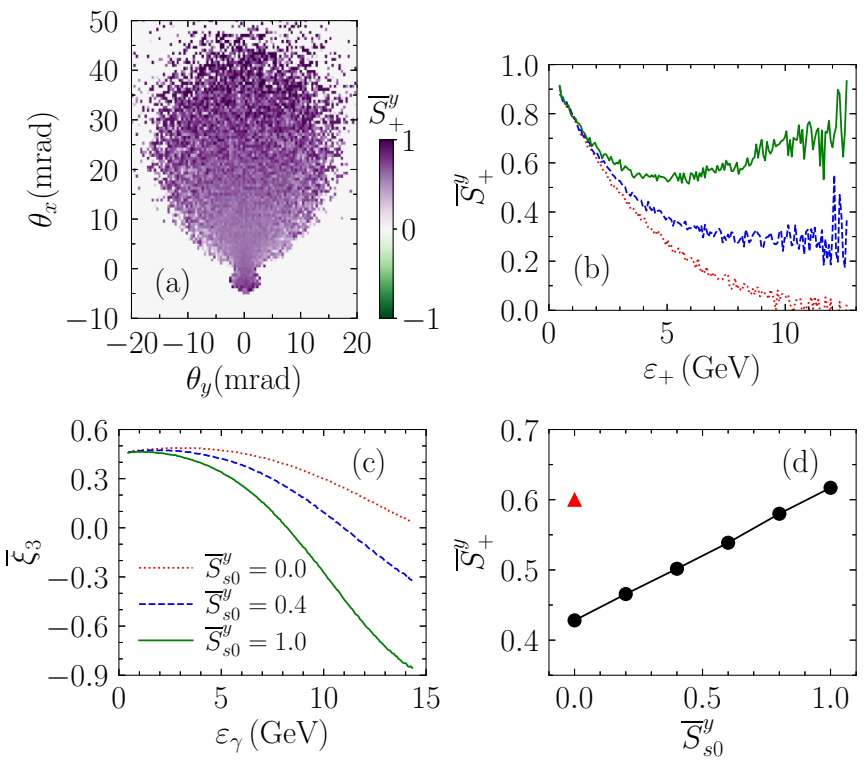

FIG. 6. (a) Transverse angular distribution of positron polarization $\bar{S}_{+}^{y}$ with an initially polarized seeding beam of $\bar{S}_{s 0}^{y}=1$. (b) $\bar{S}_{+}^{y}$ vs positron energy $\varepsilon_{+}$and (c) photon polarization $\bar{\xi}_{3}$ vs photon energy $\varepsilon_{\gamma}$ under various initial polarization $\bar{S}_{s 0}^{y}=0$ (red dotted), 0.4 (blue dashed), and 1 (green solid). (d) Polarization degree of positrons $\bar{S}_{+}^{y}$ vs $\bar{S}_{s 0}^{y}$. The red-triangle marker denotes the result excluding the effect of photon polarization. Other parameters are same as those in Fig. 2.

it is clearly visible that $\bar{S}_{+}^{y}$ has significantly improved in the small $\theta_{x}$ range, corresponding to high-energy positrons. In Fig. 6(b), we plot $\bar{S}_{+}^{y}$ with respect to $\varepsilon_{+}$under three different initial conditions of $\bar{S}_{s 0}^{y}=0,0.4$ and 1 , respectively. In the case of $\bar{S}_{s 0}^{y}=1, \bar{S}_{+}^{y}$ first decreases in the lower-energy range $\varepsilon_{+}<5 \mathrm{GeV}$, and then increases with further increasing $\varepsilon_{+}$, which is quite distinct from continuously decreasing of the $\bar{S}_{s 0}^{y}=0$ case. Hence, highly polarized positrons of high energies can be obtained. The underlying reason is originated in the photon polarization. In Fig. 6(c), we present the photon polarization $\bar{\xi}_{3}$ as a function of the photon energy $\varepsilon_{\gamma}$. For high-energy photons that more probably decay into $e^{-} e^{+}$ pairs, $\bar{\xi}_{3}$ is negative in the case of $\bar{S}_{s 0}^{y}>0$. As predicted by Fig. 3(d), these photons can generate high-energy polarized positrons of $\bar{S}_{+}^{y}>0$. Since $\bar{S}_{+}^{y}$ of low-energy positrons is also positive and insensitive to $\bar{\xi}_{3}$, the polarization degree of all positrons can be improved substantially. The dependence of the positron polarization on the initial polarization of the seeding beam is summarized in Fig. 6(d), which illustrates that $\bar{S}_{+}^{y}$ monotonically increases with $\bar{S}_{s 0}^{y}$, and $\bar{S}_{+}^{y}$ can exceed 0.6 for $\bar{S}_{s 0}^{y}=1$.

We have also performed an additional simulation to illustrate the importance of the photon polarization further. When the polarization-averaged probabilities of photon emission and pair production are employed (i.e., the emitted photons are unpolarized), the obtained $\bar{S}_{+}^{y}$ even reaches 0.6 for the $\bar{S}_{s 0}^{y}=0$ case, as shown by the red-triangle marker in Fig. 6(d), which is much larger than 0.43 including the photon polarization effect. As a result, excluding the photon polarization will significantly overestimate the final positron polarization. It is also pointed out by [56] that with the photon polarization effect included only about $30 \%$ polarization of positrons can be achieved by the two-color-laser scheme [25].

\section{CONCLUSION}

In summary, through Monte Carlo simulations, we have demonstrated that a high-energy transversely polarized positron beam with a polarization degree above $40 \%$ can be generated via multiphoton BW process in the collision of two electron beams with a proper impact parameter. Under the optimal colliding condition, the positron yield can be increased effectively while preserving a high polarization by increasing the charge density of the driving beam or the energy of the seeding beam. Note that the driving beam length should not be too small, otherwise the positron yield will decrease due to the ultrashort collision time. Utilizing the relationship of the polarization among primary electrons, emitted $\gamma$ photons, and generated positrons, the polarization degree of positrons can be improved to $60 \%$ with an initially polarized seeding beam. Such highly transversely polarized positron beams could allow detecting possible sources of $C P$ violation [57] and testing specific triple-gauge couplings in the future $e^{-} e^{+}$ linear collider.

\section{ACKNOWLEDGMENTS}

This work was supported by the National Key R\&D Program of China (Grant No. 2018YFA0404801), the National Natural Science Foundation of China (Grants No. 11775302 and No. 11827807), the Strategic Priority Research Program of Chinese Academy of Sciences (Grants No. XDA25050300, No. XDA25010300, and No. XDB16010200), and the Fundamental Research Funds for the Central Universities, the Research Funds of Renmin University of China (Grant No. 20XNLG01).
[1] D. W. Gidley, A. R. Köymen, and T. W. Capehart, Polarized Low-Energy Positrons: A New Probe of Surface Magnetism, Phys. Rev. Lett. 49, 1779 (1982).

[2] A. Kawasuso, Y. Fukaya, M. Maekawa, H. Zhang, T. Seki, T. Yoshino, E. Saitoh, and K. Takanashi, Current-induced spin polarization on a Pt surface: A new approach using spin-polarized positron annihilation spectroscopy, J. Magn. Magn. Mater. 342, 139 (2013).
[3] C. Hugenschmidt, Positrons in surface physics, Surf. Sci. Rep. 71, 547 (2016).

[4] G. Moortgat-Pick, T. Abe, G. Alexander, B. Ananthanarayan, A. Babich, V. Bharadwaj, D. Barber, A. Bartl, A. Brachmann, S. Chen et al., Polarized positrons and electrons at the linear collider, Phys. Rep. 460, 131 (2008).

[5] J. List, Polarised beams at future $e^{+} e^{-}$colliders, arXiv:2012.11267. 
[6] P. W. Zitzewitz, J. C. Van House, A. Rich, and D. W. Gidley, Spin Polarization of Low-Energy Positron Beams, Phys. Rev. Lett. 43, 1281 (1979).

[7] A. A. Sokolov and I. M. Ternov, Synchrotron Radiation (Akademie-Verlag, Berlin, 1968).

[8] V. N. Baier, Radiative polarization of electrons in storage rings, Sov. Phys. Usp. 14, 695 (1972).

[9] G. Alexander, J. Barley, Y. Batygin, S. Berridge, V. Bharadwaj, G. Bower, W. Bugg, F.-J. Decker, R. Dollan, Y. Efremenko et al., Observation of Polarized Positrons from an UndulatorBased Source, Phys. Rev. Lett. 100, 210801 (2008).

[10] T. Omori, M. Fukuda, T. Hirose, Y. Kurihara, R. Kuroda, M. Nomura, A. Ohashi, T. Okugi, K. Sakaue, T. Saito, J. Urakawa, M. Washio, and I. Yamazaki, Efficient Propagation of Polarization from Laser Photons to Positrons Through Compton Scattering and Electron-Positron Pair Creation, Phys. Rev. Lett. 96, 114801 (2006).

[11] D. Abbott, P. Adderley, A. Adeyemi, P. Aguilera, M. Ali, H. Areti, M. Baylac, J. Benesch, G. Bosson, B. Cade et al. (PEPPo Collaboration), Production of Highly Polarized Positrons using Polarized Electrons at MeV Energies, Phys. Rev. Lett. 116, 214801 (2016).

[12] H. R. Reiss, Absorption of light by light, J. Math. Phys. 3, 59 (1962).

[13] V. B. Berestetskii, E. M. Lifshitz, and L. P. Pitaevskii, Quantum Electrodynamics (Elsevier Butterworth-Heinemann, Oxford, 1982).

[14] V. N. Baier, V. Katkov, and V. M. Strakhovenko, Electromagnetic Processes at High Energies in Oriented Single Crystals (World Scientific, Singapore, 1998).

[15] V. I. Ritus, Quantum effects of the interaction of elementary particles with an intense electromagnetic field, J. Sov. Laser Res. 6, 497 (1985).

[16] A. Di Piazza, C. Müller, K. Z. Hatsagortsyan, and C. H. Keitel, Extremely high-intensity laser interactions with fundamental quantum systems, Rev. Mod. Phys. 84, 1177 (2012).

[17] E. Cartlidge, The light fantastic, Science 359, 382 (2018).

[18] C. N. Danson, C. Haefner, J. Bromage, T. Butcher, J.-C. F. Chanteloup, E. A. Chowdhury, A. Galvanauskas, L. A. Gizzi, J. Hein, D. I. Hillier et al., Petawatt and exawatt class lasers worldwide, High Power Laser Sci. Eng. 7, e54 (2019).

[19] A. R. Bell and J. G. Kirk, Possibility of Prolific Pair Production with High-Power Lasers, Phys. Rev. Lett. 101, 200403 (2008).

[20] I. V. Sokolov, N. M. Naumova, J. A. Nees, and G. A. Mourou, Pair Creation in QED-Strong Pulsed Laser Fields Interacting with Electron Beams, Phys. Rev. Lett. 105, 195005 (2010).

[21] E. N. Nerush, I. Y. Kostyukov, A. M. Fedotov, N. B. Narozhny, N. V. Elkina, and H. Ruhl, Laser Field Absorption in SelfGenerated Electron-Positron Pair Plasma, Phys. Rev. Lett. 106, 035001 (2011).

[22] G. L. Kotkin, V. G. Serbo, and V. I. Telnov, Electron (positron) beam polarization by Compton scattering on circularly polarized laser photons, Phys. Rev. ST Accel. Beams 6, 011001 (2003).

[23] D. V. Karlovets, Radiative polarization of electrons in a strong laser wave, Phys. Rev. A 84, 062116 (2011).

[24] F. Wan, R. Shaisultanov, Y.-F. Li, K. Z. Hatsagortsyan, C. H. Keitel, and J.-X. Li, Ultrarelativistic polarized positron jets via collision of electron and ultraintense laser beams, Phys. Lett. B 800, 135120 (2020).
[25] Y.-Y. Chen, P.-L. He, R. Shaisultanov, K. Z. Hatsagortsyan, and C. H. Keitel, Polarized Positron Beams Via Intense Two-Color Laser Pulses, Phys. Rev. Lett. 123, 174801 (2019).

[26] Y.-F. Li, Y.-Y. Chen, W.-M. Wang, and H.-S. Hu, Production of Highly Polarized Positron Beams Via Helicity Transfer from Polarized Electrons in a Strong Laser Field, Phys. Rev. Lett. 125, 044802 (2020).

[27] K. Yokoya and P. Chen, Beam-beam phenomena in linear colliders, in Frontiers of Particle Beams: Intensity Limitations, edited by M. Dienes, M. Month, and S. Turner (Springer, Berlin, 1992), pp. 415-445.

[28] R. J. Noble, Beamstrahlung from colliding electron-positron beams with negligible disruption, Nucl. Instrum. Methods Phys. Res., Sect. A 256, 427 (1987).

[29] P. Chen, Differential luminosity under multiphoton beamstrahlung, Phys. Rev. D 46, 1186 (1992).

[30] M. Tamburini and S. Meuren, Efficient high-energy photon production in the supercritical QED regime, arXiv:1912.07508.

[31] P. Chen and V. I. Telnov, Coherent Pair Creation in Linear Colliders, Phys. Rev. Lett. 63, 1796 (1989).

[32] F. Del Gaudio, T. Grismayer, R. A. Fonseca, W. B. Mori, and L. O. Silva, Bright $\gamma$ rays source and nonlinear Breit-Wheeler pairs in the collision of high density particle beams, Phys. Rev. Accel. Beams 22, 023402 (2019).

[33] V. Yakimenko, S. Meuren, F. Del Gaudio, C. Baumann, A. Fedotov, F. Fiuza, T. Grismayer, M. J. Hogan, A. Pukhov, L. O. Silva, and G. White, Prospect of Studying Nonperturbative QED with Beam-Beam Collisions, Phys. Rev. Lett. 122, 190404 (2019).

[34] V. Yakimenko, L. Alsberg, E. Bong, G. Bouchard, C. Clarke, C. Emma, S. Green, C. Hast, M. J. Hogan, J. Seabury, N. Lipkowitz, B. O'Shea, D. Storey, G. White, and G. Yocky, FACET-II facility for advanced accelerator experimental tests, Phys. Rev. Accel. Beams 22, 101301 (2019).

[35] Y. Jing, Y. Hao, and V. N. Litvinenko, Compensating effect of the coherent synchrotron radiation in bunch compressors, Phys. Rev. ST Accel. Beams 16, 060704 (2013).

[36] V. Yakimenko, M. Fedurin, V. Litvinenko, A. Fedotov, D. Kayran, and P. Muggli, Experimental Observation of Suppression of Coherent-Synchrotron-Radiation-Induced BeamEnergy Spread with Shielding Plates, Phys. Rev. Lett. 109, 164802 (2012).

[37] C. E. Doss, E. Adli, R. Ariniello, J. Cary, S. Corde, B. Hidding, M. J. Hogan, K. Hunt-Stone, C. Joshi, K. A. Marsh, J. B. Rosenzweig, N. Vafaei-Najafabadi, V. Yakimenko, and M. Litos, Laser-ionized, beam-driven, underdense, passive thin plasma lens, Phys. Rev. Accel. Beams 22, 111001 (2019).

[38] A. Sampath, X. Davoine, S. Corde, L. Gremillet, M. Gilljohann, M. Sangal, C. H. Keitel, R. Ariniello, J. Cary, H. Ekerfelt, C. Emma, F. Fiuza, H. Fujii, M. Hogan, C. Joshi, A. Knetsch, O. Kononenko, V. Lee, M. Litos, K. Marsh et al., Extremely Dense Gamma-Ray Pulses in Electron Beam-Multifoil Collisions, Phys. Rev. Lett. 126, 064801 (2021).

[39] N. V. Elkina, A. M. Fedotov, I. Y. Kostyukov, M. V. Legkov, N. B. Narozhny, E. N. Nerush, and H. Ruhl, QED cascades induced by circularly polarized laser fields, Phys. Rev. ST Accel. Beams 14, 054401 (2011).

[40] C. Ridgers, J. Kirk, R. Duclous, T. Blackburn, C. Brady, K. Bennett, T. Arber, and A. Bell, Modelling gamma-ray photon 
emission and pair production in high-intensity laser-matter interactions, J. Comput. Phys. 260, 273 (2014).

[41] A. Gonoskov, S. Bastrakov, E. Efimenko, A. Ilderton, M. Marklund, I. Meyerov, A. Muraviev, A. Sergeev, I. Surmin, and E. Wallin, Extended particle-in-cell schemes for physics in ultrastrong laser fields: Review and developments, Phys. Rev. E 92, 023305 (2015).

[42] K. Yokoya, User's Manual of CAIN Version 2.42.

[43] Y.-F. Li, R. Shaisultanov, Y.-Y. Chen, F. Wan, K. Z. Hatsagortsyan, C. H. Keitel, and J.-X. Li, Polarized Ultrashort Brilliant Multi-Gev $\gamma$ Rays Via Single-Shot Laser-Electron Interaction, Phys. Rev. Lett. 124, 014801 (2020).

[44] V. Bargmann, L. Michel, and V. L. Telegdi, Precession of the Polarization of Particles Moving in a Homogeneous Electromagnetic Field, Phys. Rev. Lett. 2, 435 (1959).

[45] H.-H. Song, W.-M. Wang, Y.-F. Li, B.-J. Li, Y.-T. Li, Z.-M. Sheng, L.-M. Chen, and J. Zhang, Spin and polarization effects on the nonlinear Breit-Wheeler pair production in laser-plasma interaction, New J. Phys. 23, 075005 (2021).

[46] A. Di Piazza, M. Tamburini, S. Meuren, and C. H. Keitel, Implementing nonlinear Compton scattering beyond the localconstant-field approximation, Phys. Rev. A 98, 012134 (2018).

[47] A. Di Piazza, M. Tamburini, S. Meuren, and C. H. Keitel, Improved local-constant-field approximation for strong-field QED codes, Phys. Rev. A 99, 022125 (2019).

[48] A. Ilderton, B. King, and D. Seipt, Extended locally constant field approximation for nonlinear Compton scattering, Phys. Rev. A 99, 042121 (2019).

[49] T. Podszus and A. Di Piazza, High-energy behavior of strongfield QED in an intense plane wave, Phys. Rev. D 99, 076004 (2019).
[50] A. Pukhov and J. P. Farmer, Stable Particle Acceleration in Coaxial Plasma Channels, Phys. Rev. Lett. 121, 264801 (2018).

[51] H.-H. Song, W.-M. Wang, J.-X. Li, Y.-F. Li, and Y.-T. Li, Spinpolarization effects of an ultrarelativistic electron beam in an ultraintense two-color laser pulse, Phys. Rev. A 100, 033407 (2019).

[52] J. Vieira, C.-K. Huang, W. B. Mori, and L. O. Silva, Polarized beam conditioning in plasma based acceleration, Phys. Rev. ST Accel. Beams 14, 071303 (2011).

[53] M. Wen, M. Tamburini, and C. H. Keitel, Polarized LaserWakefield-Accelerated Kiloampere Electron Beams, Phys. Rev. Lett. 122, 214801 (2019).

[54] Y. Wu, L. Ji, X. Geng, Q. Yu, N. Wang, B. Feng, Z. Guo, W. Wang, C. Qin, X. Yan, L. Zhang, J. Thomas, A. Hützen, A. Pukhov, M. Büscher, B. Shen, and R. Li, Polarized electron acceleration in beam-driven plasma wakefield based on density down-ramp injection, Phys. Rev. E 100, 043202 (2019).

[55] Z. Nie, F. Li, F. Morales, S. Patchkovskii, O. Smirnova, W. An, N. Nambu, D. Matteo, K. A. Marsh, F. Tsung, W. B. Mori, and C. Joshi, In Situ Generation of High-Energy Spin-Polarized Electrons in a Beam-Driven Plasma Wakefield Accelerator, Phys. Rev. Lett. 126, 054801 (2021).

[56] W.-Y. Liu, K. Xue, F. Wan, M. Chen, J.-X. Li, F. Liu, S.-M. Weng, Z.-M. Sheng, and J. Zhang, Trapping and acceleration of spin-polarized positrons from $\gamma$ photon splitting in wakefields, arXiv:2011.00156.

[57] B. Ananthanarayan and S. D. Rindani, CP violation at a linear collider with transverse polarization, Phys. Rev. D 70, 036005 (2004). 